\title{
Biometric analysis of protein and oil contents of soybean genotypes in different environments
}

\author{
Josiane Isabela da Silva Rodrigues(1), Klever Márcio Antunes Arruda(1), Cosme Damião Cruz ${ }^{(1)}$, \\ Newton Deniz Piovesan ${ }^{(1)}$, Everaldo Gonçalves de Barros ${ }^{(2)}$ and Maurilio Alves Moreira ${ }^{(3)}$
}

\begin{abstract}
(1)Universidade Federal de Viçosa, Instituto de Biotecnologia Aplicada à Agropecuária, Avenida P.H. Rolfs, Campus Universitário, CEP 36570-000 Viçosa, MG, Brazil. E-mail: josianeisabela@gmail.com, kleverantunes@yahoo.com.br, cdcruz@ufv.br, piovesan@ufv.br (2)Universidade Católica de Brasília, Programa de Pós-Graduação em Ciências Genômicas e Biotecnologia, Quadra SGAN 916, Módulo B, Bloco C, Sala 213, CEP 70790-160 Brasília, DF, Brazil. E-mail: everaldodebarros@gmail.com ${ }^{(3)} / n$ memorian
\end{abstract}

\begin{abstract}
The objective of this work was to identify by biometric analyses the most stable soybean parents, with higher oil or protein contents, cultivated at different seasons and locations of the state of Minas Gerais, Brazil. Forty-nine genotypes were evaluated in the municipalities of Viçosa, Visconde do Rio Branco, and São Gotardo, in the state of Minas Gerais, from 2009 to 2011. Protein and oil contents were analyzed by infrared spectrometry using a FT-NIR analyzer. The effects of genotype, environment, and genotype $\mathrm{x}$ environment interaction were significant. The BARC- 8 soybean genotype is the best parent to increase protein contents in the progenies, followed by BR 8014887 and CS 3032PTA276-3-4. Selection for high oil content is more efficient when the crossings involve the Suprema, CD 01RR8384, and A7002 genotypes, which show high mean phenotypic values, wide adaptability, and greater stability to environmental variation.
\end{abstract}

Index terms: Glycine max, FT-NIR, genotype x environment interaction, heritability.

\section{Análise biométrica do conteúdo de proteína e óleo de genótipos de soja em diferentes ambientes}

\begin{abstract}
Resumo - O objetivo deste trabalho foi identificar, por meio de análises biométricas, os genitores parentais de soja mais estáveis, com maior teor de óleo ou de proteína, cultivados em diferentes épocas e localidades do Estado de Minas Gerais. Quarenta e nove genótipos foram avaliados nos municípios de Viçosa, Visconde do Rio Branco e São Gotardo, em Minas Gerais, de 2009 a 2011. Os conteúdos de proteína e óleo foram analisados por espectrometria no infravermelho, com uso de analisador FT-NIR. Os efeitos de genótipo, ambiente e da interação genótipo $\mathrm{x}$ ambiente foram significativos. O genótipo de soja BARC-8 é o melhor genitor para o aumento no teor proteico das progênies, seguido por BR 8014887 e CS 3032PTA276-3-4. A seleção para alto teor de óleo é mais eficiente quando os cruzamentos envolvem os genótipos Suprema, CD 01RR8384 e A7002, que apresentam altos valores de média fenotípica, adaptabilidade ampla e maior estabilidade frente à variação ambiental.
\end{abstract}

Termos para indexação: Glycine max, FT-NIR, interação genótipo x ambiente, herdabilidade.

\section{Introduction}

On average, soybean (Glycine max L.) cultivars contain $40 \%$ of protein and $20 \%$ of oil, on a dry matter basis (Boerma \& Specht, 2004). Both protein and oil contents are in part determined by additive gene action, with heritability values ranging from medium to high (Rodrigues et al., 2010; Jaureguy et al., 2011).

Protein content is most often negatively correlated with grain yield, whereas the association between oil content and grain yield is positive. Therefore, there is an inverse relationship between oil and protein content
(Proulx \& Naeve, 2009; Barbosa et al., 2011; Akond et al., 2012; Popovic et al., 2012), and this association makes it difficult to develop productive cultivars with simultaneous high contents of protein and oil.

The selection for high protein or oil content should be preceded by analyses for genotype $\mathrm{x}$ environment interaction (GxE), since seed composition is affected by environmental factors, such as temperature, water availability, and nitrogen supply, especially during the seed-filling period (Ray et al., 2008). In general, supplemental nitrogen during seed filling increases seed protein content, whereas water stress and high 
temperatures reduce both protein and oil contents in the seeds. Temperature has a more pronounced effect on oil content, but it also affects protein content (Rotundo \& Westgate, 2009).

The differential response of soybean genotypes to changes in environments is often evaluated by estimating the interaction effects of genotypes $\mathrm{x}$ locations, genotypes $\mathrm{x}$ years, genotypes $\mathrm{x}$ locations $\mathrm{x}$ years, and genotypes $\mathrm{x}$ sowing dates, although these effects are complex and the biological meaning of the contrasts is hard to explain biologically (Albrecht et al., 2008; Naeve \& Huerd, 2008; Zhe et al., 2010; Barbosa et al., 2011). After estimating the interaction effects, genotype performance against environmental variations should be evaluated by analyses of adaptability and stability, which allow for identifying cultivars with predictable or responsive behavior to environmental variations, in specific or broad conditions (Cruz \& Carneiro, 2003).

The adaptability and stability of soybean genotypes for grain yield have been profusely evaluated (Oliveira et al., 2006; Cucolotto et al., 2007; Pelúzio et al., 2008; Kamaluddin \& Ahmad, 2012). However, few of these analyses were performed for protein and oil contents. Brazilian studies on this regard often assessed a very small number of cultivars, with low protein or oil content, bringing small contribution to the breeding programs.

The objective of this work was to identify by biometric analyses the most stable soybean parents, with higher oil or protein contents, cultivated at different seasons and locations of the state of Minas Gerais, Brazil.

\section{Materials and Methods}

Forty-nine soybean genotypes from the breeding program for soybean quality of Universidade Federal de Viçosa were evaluated in three different regions of the state of Minas Gerais, Brazil, between 2009 and 2011. The first experiment was carried out in December 2009 , at the municipality of Viçosa $\left(20^{\circ} 45^{\prime} \mathrm{S}, 42^{\circ} 52^{\prime} \mathrm{W}\right.$, at $663 \mathrm{~m}$ altitude); the next two, in February 2010, at the municipalities of Visconde do Rio Branco $\left(21^{\circ} 00^{\prime} \mathrm{S}\right.$, $42^{\circ} 50^{\prime} \mathrm{W}$, at $358 \mathrm{~m}$ altitude) and São Gotardo $\left(19^{\circ} 18^{\prime} \mathrm{S}\right.$, $46^{\circ} 02^{\prime} \mathrm{W}$, at $1,152 \mathrm{~m}$ altitude); and the last one, in October 2011, also at São Gotardo.
The predominant climate in these locations, according to Köppen's classification, is of the Cwa type, or mesothermal humid, characterized by hot, humid summers and cool, dry winters, with annual mean rainfall of $1,200 \mathrm{~mm}$ and maximum and minimum mean temperatures of 26.1 and $14.0^{\circ} \mathrm{C}$, respectively (Silva et al., 2004).

All experiments were carried out under a randomized complete block design, with three replicates. Fifteen seeds were sown per meter, with $0.5 \mathrm{~m}$ spacing between rows. After manual harvest, the grains were ground in an industrial mill, model MA020 (Marconi Equipamentos para Laboratório, Piracicaba, SP, Brazil). Soybean flours were analyzed for protein and oil contents by infrared spectrometry, using the Antaris II FT-NIR analyzer (Thermo Fisher Scientific Brasil Instrumentos de Processo Ltda., São Paulo, SP, Brazil).

A joint analysis of the experiments was done, with phenotypic data subjected to analysis of variance based on factorial arrangement, to test the effects of genotype, environment, and genotype $\mathrm{x}$ environment interaction, using the statistical model: $\mathrm{Y}_{\mathrm{ijk}}=\mu+\mathrm{G}_{\mathrm{i}}+\mathrm{E}_{\mathrm{j}}+\mathrm{GE}_{\mathrm{ij}}+\mathrm{B} /$ $\mathrm{E}_{\mathrm{jk}}+\varepsilon_{\mathrm{ijk}}$. The effects of genotypes $\left(\mathrm{G}_{\mathrm{i}}\right)$ were considered fixed, and the ones of environments $\left(\mathrm{E}_{\mathrm{ijk}}\right)$, random. The variance components were estimated as: $\hat{\emptyset}_{\mathrm{g}}=(\mathrm{MSG}-\mathrm{MSGE}) / \mathrm{Er}$ and $\delta_{\mathrm{ge}}^{2}=(\mathrm{MSGE}-\mathrm{MSR}) / \mathrm{rL}$, in which MSG is the mean square of genotypes; MSGE is the mean square of the GxE interaction; MSR is the mean square of the residue; $E$ is the number of environments; $r$ is the number of replicates; and $\mathrm{L}=\mathrm{g}(\mathrm{g}-1)$, given that $\mathrm{g}$ is the number of genotypes.

After the joint analysis of the experiments, analyses of environmental stratification and of adaptability and stability of genotypes were performed according to the following methods: Wricke (1965), Annicchiarico (1992), Lin \& Binns (1988) modified by Cruz \& Carneiro (2003), and centroid (Nascimento et al., 2009). The proportions of simple and complex mean squares for the interaction between genotypes and pairs of environments (MSGxEjj') were calculated according to Robertson (1959) and Cruz \& Castoldi (1991). All analyses were performed using the Genes software (Cruz, 2006).

\section{Results and Discussion}

Analysis of variance revealed a significant effect of genotypes on their protein and oil contents. The averages of the experiments varied from 18.75 to 
$20.23 \%$ for oil content, and from 38.12 to $41.14 \%$ for protein content. The averages of the four evaluations varied from 14.40 to $24.20 \%$ for oil content, and from 32.07 to $48.57 \%$ for protein content, which are amplitudes close to the ones reported by Albrecht et al. (2008) and Shi et al. (2010).

Considering the general mean values, the highest oil percentage was obtained with the Suprema genotype (23.01\%), followed by CD 01RR8384 (22.91\%). The lowest value was observed with CS 3032PTA137-4-10 $(16.48 \%)$. For protein content, the highest value was obtained with the BARC-8 genotype (45.18\%), followed by BR 8014887 (44.71\%). The Suprema genotype had the lowest percentage (34.25\%).

Broad heritability values were high in the four evaluations, ranging from 0.90 to 0.95 for oil content, and from 0.86 to 0.96 for protein content. Oil and protein contents were negatively correlated, which agrees with the literature (Liang et al., 2010; Rodrigues et al., 2010). These correlations were high, with small variation $(\mathrm{r}=-0.80 * *$ to $-0.92 * *)$, revealing the degree of genetic association between the characters and explaining the difficulties for simultaneous selection.

The coefficients of variation were below $5 \%$, which reveals good precision. Based on the ratio between the highest and the lowest residual mean square, the residual variances of the experiments were considered homogeneous.

The effects of genotype, environment, and GxE interaction were significant at $1 \%$ probability, for both traits (Table 1). The interaction was predominantly complex for both traits, in all pairs of environments.

The environmental effect had greater contribution to the variation in the characteristics, compared to the

Table 1. Analysis of variance for oil and protein contents of 49 soybean (Glycine max) genotypes, cultivated at different seasons and regions of the state of Minas Gerais, Brazil ${ }^{(1)}$.

\begin{tabular}{|c|c|c|c|}
\hline \multirow{2}{*}{ Source of variation } & \multirow{2}{*}{$\mathrm{df}$} & \multicolumn{2}{|c|}{ Mean squares } \\
\hline & & Protein $(\%)$ & Oil (\%) \\
\hline Blocks/Environments & 8 & 11.73 & 8.31 \\
\hline Genotypes & 48 & $89.22 * *$ & $30.39 * *$ \\
\hline Environments & 3 & $236.44 * *$ & $63.53 * *$ \\
\hline Genotypes x Environments & 144 & $6.56 * *$ & $3.80 * *$ \\
\hline Error & 384 & 2 & 0.82 \\
\hline Mean & - & 39.67 & 19.51 \\
\hline Coefficient of variation $(\%)$ & - & 3.56 & 4.64 \\
\hline Relation higher/lower MSR & - & 3.77 & 3.5 \\
\hline $\mathrm{CVg} / \mathrm{CVe}$ & - & 1.86 & 1.64 \\
\hline
\end{tabular}

other main effects. The protein contents were higher in the experiment sown in December, and had similar values in the experiments sown in February and October. The oil content was higher in the experiment sown in October. This result can be attributed to the generally higher temperatures observed in this season, which positively influence oil accumulation. Barbosa et al. (2011) found that higher temperatures during grain filling favored oil accumulation and reported reduced oil contents in late sowings.

In the environmental stratification analysis, no group of environments showed nonsignificant GxE interaction. This result may be explained by the magnitude of the GxE interactions, which was high enough to prevent the absence of a similar response pattern of the genotypes in the pairs of environments (Cruz, 2006).

Based on the estimates of the stability parameter of Wricke (1965), the most stable genotypes in relation to oil and protein contents were Garantia (19.74\% oil) and CD 226RR (39.62\% protein), respectively (Table 2). However, these genotypes did not stand out in their oil and protein contents. The following soybean genotypes were stable and had higher protein contents: CS 3032PTA276-3-4 (44.31\% protein), B3PTA382-2-10 (43.80\%), and CS 3032PTA137-4-10 (43.55\%). None of the genotypes with great stability stood out as to oil content, except for CD 219RR (21.61\% oil).

Using the recommendation index proposed by Annicchiarico (1992), the BARC-8, BR 8014887, and CS 3032PTA276-3-4 genotypes had greater general stability for protein content. For oil content, the highest stabilities were shown by the CD 01RR8384, Suprema, and A7002 genotypes (Table 2).

For the Lin \& Binns (1988) method modified by Cruz \& Carneiro (2003), the BARC-8, BR 8014887, and CS 3032PTA276-3-4 genotypes had greater adaptability and stability for protein content, considering all environments (Table 3). Similar results were obtained with the Annicchiarico (1992) method (Table 2). For oil content, the CD 01RR8384, Suprema, and A7002 genotypes had greater adaptability and stability in all environments (Table 3 ).

The centroid method (Table 4) assumes that the genotype with highest general adaptability is the one that shows maximum values for the variable in analysis, both in favorable and unfavorable environments; whereas the genotype with minimum values has little 
Table 2. Stability estimates of soybean (Glycine max) genotypes cultivated at different seasons and regions of the state of Minas Gerais, Brazil, according to the methods of Wricke (1965) and Annicchiarico (1992)(1).

\begin{tabular}{|c|c|c|c|c|c|c|c|c|}
\hline \multirow[t]{3}{*}{ Genotype } & \multicolumn{2}{|c|}{ Wricke (1965) } & \multicolumn{6}{|c|}{ Annicchiarico (1992) } \\
\hline & & & \multicolumn{3}{|c|}{ Protein } & \multicolumn{3}{|c|}{ Oil } \\
\hline & Protein & Oil & General & Favorable & Unfavorable & General & Favorable & Unfavorable \\
\hline 1. Garantia & 0.38 & 0.07 & 97.44 & 96.77 & 98.22 & 100.88 & 100.31 & 101.67 \\
\hline 2. Tucunaré & 2.59 & 2.57 & 93.41 & 96.39 & 90.76 & 104.88 & 111.09 & 100.44 \\
\hline 3. Luziânia & 0.45 & 0.85 & 98.20 & 99.14 & 97.51 & 98.45 & 101.93 & 95.94 \\
\hline 4. UFV 16 & 2.18 & 1.75 & 95.84 & 96.74 & 94.87 & 100.20 & 101.04 & 98.99 \\
\hline 5. CS 3032PTA182 & 1.13 & 3.16 & 104.68 & 106.10 & 103.26 & 89.73 & 95.58 & 85.12 \\
\hline 6. Sambaíba & 2.09 & 0.29 & 93.55 & 94.57 & 92.21 & 99.83 & 100.04 & 99.63 \\
\hline 7. CS 94731 & 4.00 & 2.98 & 101.08 & 105.03 & 97.71 & 91.57 & 97.66 & 86.86 \\
\hline 8. M-SOY 8914 & 1.08 & 2.86 & 93.13 & 94.81 & 91.89 & 103.49 & 110.00 & 98.79 \\
\hline 9. UFV 18 & 2.78 & 1.88 & 93.98 & 93.41 & 95.09 & 103.15 & 104.87 & 101.48 \\
\hline 10. M-SOY 8400 & 3.10 & 3.09 & 97.78 & 95.59 & 100.52 & 94.50 & 92.48 & 96.11 \\
\hline 11. DM339 & 5.29 & 3.00 & 97.38 & 96.76 & 98.52 & 94.38 & 91.14 & 97.88 \\
\hline 12. UFVS 2012 & 1.55 & 1.12 & 92.78 & 92.63 & 92.80 & 105.13 & 106.03 & 103.97 \\
\hline 13. UFVS 2011 & 0.88 & 1.65 & 94.80 & 95.28 & 94.08 & 99.00 & 100.94 & 97.30 \\
\hline 14. M-SOY 8001 & 1.13 & 1.68 & 92.99 & 93.09 & 92.59 & 100.14 & 103.38 & 97.10 \\
\hline 15. UFVS 2001 & 1.48 & 0.52 & 99.24 & 97.54 & 101.33 & 97.13 & 98.10 & 95.99 \\
\hline 16. Conquista & 0.40 & 0.22 & 96.63 & 97.97 & 95.52 & 99.26 & 99.51 & 99.20 \\
\hline 17. Suprema & 3.54 & 2.06 & 85.01 & 83.43 & 86.41 & 116.31 & 115.64 & 116.84 \\
\hline 18. M-SOY 6101 & 1.20 & 2.43 & 89.87 & 88.63 & 91.32 & 110.57 & 107.36 & 115.37 \\
\hline 19. A7002 & 1.78 & 0.78 & 92.98 & 94.26 & 91.51 & 114.90 & 114.39 & 115.11 \\
\hline 20. PI 417360 & 18.81 & 22.12 & 100.55 & 93.39 & 108.84 & 83.35 & 76.80 & 90.32 \\
\hline 21. CS 3032PTA190-5-1 & 0.81 & 1.01 & 109.31 & 108.49 & 109.90 & 87.49 & 89.87 & 85.20 \\
\hline 22. CS 3032PTA137-4-10 & 0.41 & 2.48 & 109.32 & 109.71 & 108.89 & 82.58 & 87.53 & 78.35 \\
\hline 23. В3РТA382-2-10 & 0.16 & 0.30 & 110.12 & 109.84 & 110.53 & 89.79 & 91.80 & 88.13 \\
\hline 24. CS 3032PTA276-3-4 & 0.05 & 0.38 & 111.48 & 111.12 & 111.84 & 85.58 & 87.25 & 84.06 \\
\hline 25. В3РТА216-1-9 & 1.04 & 0.84 & 108.87 & 108.93 & 108.54 & 93.71 & 92.05 & 95.27 \\
\hline 26. BARC-8 & 2.47 & 1.04 & 112.79 & 111.63 & 113.92 & 92.41 & 94.15 & 90.99 \\
\hline 27. CS 3032PTA276-1-2 & 0.43 & 0.57 & 109.79 & 109.19 & 110.36 & 86.90 & 88.97 & 85.18 \\
\hline 28. CS 3032PTA167-1-2 & 1.73 & 1.81 & 103.44 & 103.74 & 103.07 & 92.85 & 94.96 & 90.93 \\
\hline 29. B3РТA213-3-4 & 1.27 & 0.97 & 108.18 & 110.01 & 106.79 & 93.74 & 93.16 & 93.91 \\
\hline 30. CS 303TNKCA & 3.20 & 0.49 & 95.38 & 95.76 & 94.50 & 106.11 & 108.14 & 104.39 \\
\hline 31. Vencedora & 3.60 & 1.12 & 93.02 & 97.44 & 89.51 & 100.20 & 102.88 & 98.24 \\
\hline 32. PI 181544 & 3.35 & 8.79 & 103.85 & 104.71 & 102.77 & 90.40 & 88.65 & 90.77 \\
\hline 33. PI 371611 & 1.48 & 2.60 & 97.79 & 95.83 & 100.22 & 104.11 & 102.10 & 106.75 \\
\hline 34. PI 371610 & 0.80 & 3.10 & 95.34 & 95.15 & 95.39 & 108.28 & 104.53 & 112.02 \\
\hline 35. CD 225RR & 0.53 & 0.78 & 98.94 & 99.61 & 98.17 & 102.63 & 101.60 & 103.40 \\
\hline 36. CD 224 & 2.99 & 2.15 & 91.93 & 88.87 & 96.55 & 104.59 & 101.06 & 110.23 \\
\hline 37. CD 219RR & 0.46 & 0.31 & 91.80 & 91.55 & 91.88 & 110.10 & 108.86 & 111.38 \\
\hline 38. PI 235347 & 3.67 & 3.42 & 98.19 & 102.42 & 94.86 & 97.65 & 100.53 & 94.40 \\
\hline 39. CD 226RR & 0.04 & 1.44 & 99.70 & 99.61 & 99.75 & 101.88 & 99.21 & 104.94 \\
\hline 40. UFV 20 & 3.16 & 1.65 & 93.22 & 96.17 & 91.36 & 99.26 & 103.32 & 95.97 \\
\hline 41. CD 222 & 1.69 & 1.06 & 89.74 & 93.54 & 87.16 & 104.51 & 107.46 & 101.95 \\
\hline 42. CD 01 RR8376 & 1.38 & 0.57 & 92.78 & 92.82 & 92.47 & 108.97 & 106.63 & 112.34 \\
\hline 43. CD 983321RR & 0.58 & 0.48 & 98.17 & 97.09 & 99.48 & 100.95 & 99.15 & 103.97 \\
\hline 44. BR 8014887 & 1.67 & 1.01 & 111.78 & 110.46 & 112.86 & 87.52 & 86.20 & 89.12 \\
\hline 45. CD 01RR8384 & 1.71 & 0.69 & 92.01 & 90.54 & 93.46 & 116.45 & 114.10 & 120.54 \\
\hline 46. Monarca & 0.93 & 2.24 & 98.34 & 97.47 & 99.01 & 97.94 & 96.24 & 99.13 \\
\hline 47. UFVTN-105AP & 1.79 & 0.89 & 107.72 & 107.09 & 108.18 & 88.30 & 91.29 & 85.80 \\
\hline 48. CD 201 & 0.61 & 1.12 & 102.93 & 102.22 & 104.05 & 98.10 & 95.77 & 100.64 \\
\hline 49. CD 2013PTA & 2.14 & 1.60 & 108.27 & 106.20 & 110.45 & 92.57 & 89.17 & 97.65 \\
\hline
\end{tabular}

${ }^{(1)}$ Genotypes associated with lower values for the Wricke (1965) method and with higher values for the Annicchiarico (1992) method are more stable. 
Table 3. Stability estimates of soybean (Glycine max) genotypes cultivated at different seasons and regions of the state of Minas Gerais, Brazil, according to the method of Lin \& Binns (1988) modified by Cruz \& Carneiro (2003) ${ }^{(1)}$.

\begin{tabular}{|c|c|c|c|c|c|c|c|c|c|}
\hline \multirow[t]{2}{*}{ Genotype } & \multicolumn{3}{|c|}{ Protein } & \multicolumn{3}{|c|}{ Oil } & \multicolumn{3}{|c|}{ Multivariate analysis } \\
\hline & General & Favorable & Unfavorable & General & Favorable & Unfavorable & General & Favorable & Unfavorable \\
\hline 1. Garantia & 13.06 & 11.11 & 15.01 & 2.79 & 2.67 & 2.91 & 0.24 & 0.33 & 0.22 \\
\hline 2. Tucunaré & 18.09 & 11.31 & 24.86 & 1.96 & 0.80 & 3.12 & 0.26 & 0.35 & 0.31 \\
\hline 3. Luziânia & 12.52 & 9.34 & 15.69 & 3.36 & 2.31 & 4.42 & 0.25 & 0.37 & 0.27 \\
\hline 4. UFV 16 & 15.02 & 10.87 & 19.17 & 2.59 & 2.21 & 2.97 & 0.25 & 0.35 & 0.26 \\
\hline 5. CS 3032PTA 182 & 7.23 & 4.53 & 9.93 & 5.91 & 3.79 & 8.02 & 0.29 & 0.49 & 0.34 \\
\hline 6. Sambaíba & 17.81 & 12.80 & 22.83 & 3.04 & 2.69 & 3.39 & 0.30 & 0.41 & 0.31 \\
\hline 7. CS 94731 & 10.55 & 5.19 & 15.91 & 5.18 & 2.94 & 7.43 & 0.29 & 0.49 & 0.37 \\
\hline 8. M-SOY 8914 & 17.97 & 12.71 & 23.22 & 2.25 & 0.96 & 3.53 & 0.27 & 0.35 & 0.31 \\
\hline 9. UFV 18 & 15.06 & 14.90 & 15.22 & 2.25 & 1.77 & 2.73 & 0.24 & 0.29 & 0.22 \\
\hline 10. M-SOY 8400 & 11.29 & 12.39 & 10.19 & 4.11 & 4.58 & 3.63 & 0.26 & 0.38 & 0.20 \\
\hline 11. DM339 & 11.06 & 11.61 & 10.51 & 4.38 & 5.01 & 3.75 & 0.27 & 0.41 & 0.21 \\
\hline 12. UFVS 2012 & 18.14 & 14.34 & 21.94 & 1.84 & 1.38 & 2.30 & 0.26 & 0.32 & 0.26 \\
\hline 13. UFVS 2011 & 16.37 & 12.54 & 20.21 & 2.91 & 2.47 & 3.35 & 0.28 & 0.38 & 0.28 \\
\hline 14. M-SOY 8001 & 18.21 & 14.20 & 22.22 & 2.90 & 1.80 & 3.99 & 0.29 & 0.39 & 0.32 \\
\hline 15. UFVS 2001 & 11.01 & 10.28 & 11.74 & 3.64 & 3.13 & 4.16 & 0.25 & 0.36 & 0.23 \\
\hline 16. Conquista & 14.46 & 10.57 & 18.34 & 3.08 & 2.57 & 3.58 & 0.26 & 0.37 & 0.27 \\
\hline 17. Suprema & 27.24 & 24.79 & 29.70 & 0.39 & 0.37 & 0.40 & 0.30 & 0.29 & 0.27 \\
\hline 18. M-SOY 6101 & 21.50 & 19.06 & 23.94 & 0.87 & 1.28 & 0.45 & 0.26 & 0.27 & 0.22 \\
\hline 19. A7002 & 17.05 & 13.50 & 20.60 & 0.51 & 0.50 & 0.51 & 0.20 & 0.22 & 0.20 \\
\hline 20. PI 417360 & 8.18 & 12.63 & 3.74 & 8.38 & 10.10 & 6.64 & 0.38 & 0.64 & 0.24 \\
\hline 21. CS 3032PTA190-5-1 & 3.52 & 3.24 & 3.81 & 6.74 & 5.39 & 8.08 & 0.28 & 0.50 & 0.28 \\
\hline 22. CS 3032PTA137-4-10 & 4.07 & 2.77 & 5.37 & 8.41 & 5.84 & 10.99 & 0.34 & 0.62 & 0.39 \\
\hline 23. В3РТA382-2-10 & 3.63 & 2.94 & 4.33 & 5.75 & 4.68 & 6.83 & 0.24 & 0.43 & 0.25 \\
\hline 24. CS 3032PTA276-3-4 & 2.86 & 2.29 & 3.43 & 7.45 & 6.24 & 8.67 & 0.29 & 0.55 & 0.30 \\
\hline 25. В3РTA216-1-9 & 4.33 & 2.99 & 5.66 & 4.40 & 4.17 & 4.63 & 0.20 & 0.36 & 0.19 \\
\hline 26. BARC- 8 & 2.08 & 1.98 & 2.17 & 4.73 & 4.18 & 5.27 & 0.19 & 0.35 & 0.18 \\
\hline 27. CS 3032PTA276-1-2 & 3.54 & 2.93 & 4.16 & 6.90 & 5.69 & 8.12 & 0.28 & 0.51 & 0.29 \\
\hline 28. CS 3032PTA167-1-2 & 7.80 & 5.55 & 10.06 & 4.87 & 3.85 & 5.89 & 0.25 & 0.42 & 0.27 \\
\hline 29. B3РTA213-3-4 & 4.74 & 2.59 & 6.90 & 4.42 & 3.83 & 5.01 & 0.21 & 0.37 & 0.22 \\
\hline 30. CS 303TNKCA & 15.71 & 11.56 & 19.86 & 1.71 & 1.19 & 2.23 & 0.23 & 0.29 & 0.24 \\
\hline 31. Vencedora & 18.88 & 10.96 & 26.80 & 2.76 & 1.80 & 3.72 & 0.30 & 0.42 & 0.35 \\
\hline 32. PI 181544 & 6.36 & 5.39 & 7.33 & 5.42 & 5.90 & 4.94 & 0.26 & 0.45 & 0.22 \\
\hline 33. PI 371611 & 12.29 & 11.82 & 12.77 & 1.79 & 2.14 & 1.44 & 0.19 & 0.24 & 0.16 \\
\hline 34. PI 371610 & 15.51 & 12.51 & 18.51 & 1.06 & 1.34 & 0.78 & 0.20 & 0.24 & 0.19 \\
\hline 35. CD 225RR & 11.49 & 8.99 & 13.98 & 2.16 & 2.11 & 2.21 & 0.20 & 0.27 & 0.19 \\
\hline 36. CD 224 & 18.08 & 19.17 & 16.98 & 1.75 & 2.42 & 1.07 & 0.25 & 0.28 & 0.18 \\
\hline 37. CD 219RR & 19.78 & 16.34 & 23.21 & 0.99 & 0.95 & 1.03 & 0.24 & 0.27 & 0.24 \\
\hline 38. PI 235347 & 13.03 & 6.85 & 19.22 & 3.09 & 2.05 & 4.14 & 0.25 & 0.38 & 0.30 \\
\hline 39. CD 226RR & 11.32 & 9.24 & 13.41 & 2.25 & 2.33 & 2.18 & 0.20 & 0.28 & 0.19 \\
\hline 40. UFV 20 & 17.65 & 11.41 & 23.90 & 2.91 & 2.02 & 3.81 & 0.29 & 0.41 & 0.33 \\
\hline 41. CD 222 & 22.51 & 14.63 & 30.39 & 1.90 & 1.32 & 2.48 & 0.30 & 0.40 & 0.34 \\
\hline 42. CD 01RR8376 & 17.21 & 15.00 & 19.42 & 1.18 & 1.45 & 0.91 & 0.22 & 0.26 & 0.20 \\
\hline 43. CD 983321RR & 11.95 & 11.20 & 12.70 & 2.64 & 2.88 & 2.40 & 0.22 & 0.30 & 0.19 \\
\hline 44. BR 8014887 & 2.36 & 2.40 & 2.31 & 6.26 & 6.41 & 6.10 & 0.25 & 0.46 & 0.21 \\
\hline 45. CD 01RR8384 & 17.97 & 17.28 & 18.66 & 0.31 & 0.49 & 0.14 & 0.20 & 0.19 & 0.17 \\
\hline 46. Monarca & 11.63 & 10.35 & 12.91 & 3.51 & 3.58 & 3.44 & 0.25 & 0.36 & 0.22 \\
\hline 47. UFVTN-105AP & 5.04 & 3.99 & 6.10 & 6.37 & 4.89 & 7.85 & 0.28 & 0.49 & 0.30 \\
\hline 48. CD 201 & 8.27 & 7.34 & 9.20 & 3.22 & 3.36 & 3.09 & 0.20 & 0.31 & 0.18 \\
\hline 49. CD 2013PTA & 3.78 & 4.32 & 3.23 & 4.60 & 5.30 & 3.91 & 0.20 & 0.36 & 0.15 \\
\hline
\end{tabular}

${ }^{(1)}$ Lower values indicate greater adaptability and stability. General, estimates determined in all environments; favorable, estimates in favorable environments; unfavorable, estimates in unfavorable environments. 
Table 4. Adaptability classification of soybean (Glycine max) genotypes cultivated at different seasons and regions of the state of Minas Gerais, Brazil, according to the centroid method (Nascimento et al., 2009)(1).

\begin{tabular}{|c|c|c|c|c|c|c|}
\hline \multirow[t]{2}{*}{ Genotypes } & \multicolumn{3}{|c|}{ Protein } & \multicolumn{3}{|c|}{ Oil } \\
\hline & Mean & Classification & Probability $^{(2)}$ & Mean & Classification & Probability \\
\hline 1. Garantia & 38.82 & $\mathrm{~V}$ & 0.48 & 19.74 & $\mathrm{~V}$ & 0.66 \\
\hline 2. Tucunaré & 37.56 & $\mathrm{~V}$ & 0.29 & 20.82 & VII & 0.33 \\
\hline 3. Luziânia & 39.16 & V & 0.54 & 19.42 & $\mathrm{~V}$ & 0.48 \\
\hline 4. UFV 16 & 38.47 & V & 0.35 & 19.85 & $\mathrm{~V}$ & 0.38 \\
\hline 5. CS 3032PTA182 & 41.84 & $\mathrm{~V}$ & 0.25 & 17.91 & $\mathrm{~V}$ & 0.25 \\
\hline 6. Sambaíba & 37.55 & V & 0.29 & 19.60 & $\mathrm{~V}$ & 0.61 \\
\hline 7. CS 94731 & 40.71 & V & 0.30 & 18.27 & $\mathrm{~V}$ & 0.28 \\
\hline 8. M-SOY 8914 & 37.26 & $\mathrm{~V}$ & 0.28 & 20.57 & VII & 0.29 \\
\hline 9. UFV 18 & 37.72 & $\mathrm{~V}$ & 0.29 & 20.43 & $\mathrm{~V}$ & 0.28 \\
\hline 10. M-SOY 8400 & 39.26 & V & 0.36 & 18.81 & V & 0.32 \\
\hline 11. DM339 & 39.26 & $\mathrm{~V}$ & 0.31 & 18.76 & $\mathrm{~V}$ & 0.31 \\
\hline 12. UFVS 2012 & 37.15 & $\mathrm{~V}$ & 0.27 & 20.74 & $\mathrm{~V}$ & 0.27 \\
\hline 13. UFVS 2011 & 37.90 & $\mathrm{~V}$ & 0.33 & 19.61 & V & 0.41 \\
\hline 14. M-SOY 8001 & 37.19 & $\mathrm{~V}$ & 0.28 & 19.83 & $\mathrm{~V}$ & 0.38 \\
\hline 15. UFVS 2001 & 39.71 & $\mathrm{~V}$ & 0.45 & 19.12 & $\mathrm{~V}$ & 0.49 \\
\hline 16. Conquista & 38.54 & $\mathrm{~V}$ & 0.43 & 19.47 & $\mathrm{~V}$ & 0.65 \\
\hline 17. Suprema & 34.25 & IV & 0.63 & 23.01 & $\mathrm{I}$ & 0.48 \\
\hline 18. M-SOY 6101 & 35.96 & IV & 0.32 & 21.90 & VI & 0.28 \\
\hline 19. A7002 & 37.27 & $\mathrm{~V}$ & 0.27 & 22.61 & I & 0.37 \\
\hline 20. PI 417360 & 41.01 & VII & 0.26 & 17.19 & IV & 0.24 \\
\hline 21. CS 3032PTA190-5-1 & 43.63 & $\mathrm{I}$ & 0.25 & 17.30 & IV & 0.25 \\
\hline 22. CS 3032PTA137-4-10 & 43.55 & I & 0.25 & 16.48 & IV & 0.31 \\
\hline 23. В3РTA382-2-10 & 43.80 & I & 0.28 & 17.67 & $\mathrm{~V}$ & 0.27 \\
\hline 24. CS 3032PTA276-3-4 & 44.31 & I & 0.34 & 16.84 & IV & 0.31 \\
\hline 25. В3РТА216-1-9 & 43.48 & I & 0.23 & 18.47 & $\mathrm{~V}$ & 0.35 \\
\hline 26. BARC-8 & 45.18 & I & 0.55 & 18.27 & $\mathrm{~V}$ & 0.32 \\
\hline 27. CS 3032PTA276-1-2 & 43.75 & I & 0.26 & 17.13 & IV & 0.26 \\
\hline 28. CS 3032PTA167-1-2 & 41.40 & $\mathrm{~V}$ & 0.29 & 18.41 & $\mathrm{~V}$ & 0.31 \\
\hline 29. В3РТА213-3-4 & 43.23 & VI & 0.22 & 18.50 & $\mathrm{~V}$ & 0.35 \\
\hline 30. CS 303TNKCA & 38.36 & $\mathrm{~V}$ & 0.33 & 20.86 & $\mathrm{~V}$ & 0.26 \\
\hline 31. Vencedora & 37.51 & $\mathrm{~V}$ & 0.27 & 19.79 & $\mathrm{~V}$ & 0.43 \\
\hline 32. PI 181544 & 41.72 & $\mathrm{~V}$ & 0.23 & 18.26 & $\mathrm{~V}$ & 0.23 \\
\hline 33. PI 371611 & 39.13 & $\mathrm{~V}$ & 0.43 & 20.66 & $\mathrm{~V}$ & 0.25 \\
\hline 34. PI 371610 & 38.09 & $\mathrm{~V}$ & 0.35 & 21.50 & VI & 0.23 \\
\hline 35. CD 225RR & 39.46 & V & 0.57 & 20.22 & $\mathrm{~V}$ & 0.38 \\
\hline 36. CD 224 & 36.91 & $\mathrm{~V}$ & 0.24 & 20.71 & $\mathrm{~V}$ & 0.24 \\
\hline 37. CD 219RR & 36.61 & $\mathrm{~V}$ & 0.24 & 21.61 & VII & 0.20 \\
\hline 38. PI 235347 & 39.54 & $\mathrm{~V}$ & 0.34 & 19.48 & $\mathrm{~V}$ & 0.33 \\
\hline 39. CD 226RR & 39.62 & $\mathrm{~V}$ & 0.82 & 20.13 & $\mathrm{~V}$ & 0.36 \\
\hline 40. UFV 20 & 37.53 & $\mathrm{~V}$ & 0.27 & 19.67 & $\mathrm{~V}$ & 0.40 \\
\hline 41. CD 222 & 36.04 & IV & 0.26 & 20.63 & $\mathrm{~V}$ & 0.27 \\
\hline 42. CD 01RR8376 & 37.13 & $\mathrm{~V}$ & 0.26 & 21.42 & VI & 0.21 \\
\hline 43. CD 983321RR & 39.15 & $\mathrm{~V}$ & 0.51 & 19.84 & $\mathrm{~V}$ & 0.50 \\
\hline 44. BR 8014887 & 44.71 & I & 0.45 & 17.28 & $\mathrm{~V}$ & 0.23 \\
\hline 45. CD 01RR8384 & 36.85 & $\mathrm{~V}$ & 0.24 & 22.91 & I & 0.46 \\
\hline 46. Monarca & 39.27 & $\mathrm{~V}$ & 0.49 & 19.41 & $\mathrm{~V}$ & 0.37 \\
\hline 47. UFVTN-105AP & 43.11 & VII & 0.22 & 17.45 & $\mathrm{~V}$ & 0.24 \\
\hline 48. CD 201 & 41.06 & V & 0.34 & 19.36 & $\mathrm{~V}$ & 0.46 \\
\hline 49. CD 2013PTA & 43.36 & VII & 0.26 & 18.31 & $\mathrm{~V}$ & 0.31 \\
\hline
\end{tabular}

${ }^{(1)}$ Classification of the genotypes: I, high general adaptability; II, specific adaptability to favorable environments; III, specific adaptability to unfavorable environments; IV, little adaptability; V, mean general adaptability; VI, mean specific adaptability to favorable environments; VII, mean specific adaptability to unfavorable environments. ${ }^{(2)}$ Probability associated to the classification of the soybean genotypes. 
adaptability. For protein content, eight genotypes were classified with high general adaptability: BARC-8, BR 8014887, CS 3032PTA276-3-4, B3PTA382-2-10, CS 3032PTA276-1-2, CS 3032PTA190-5-1, CS 3032PTA137-4-10, and B3PTA216-1-9, also indicated by the methods of Annicchiarico (1992) and of Lin \& Binns (1988) modified by Cruz \& Carneiro (2003). In addition to greater stability and general adaptability, the BARC- 8 and BR 8014887 genotypes had the highest mean percentage of protein. The other genotypes with high general adaptability by the centroid method also had high protein contents, above $43 \%$. However, the CD 222, M-SOY 6101, and Suprema genotypes were poorly adapted for protein content.

For oil content, only the Suprema, CD 01RR8384, and A7002 genotypes had high general adaptability by the centroid method (Table 4). These genotypes were also indicated by the methods of Annicchiarico (1992) and Lin \& Binns (1988). The CS 3032PTA190-5-1, PI 417360, CS 3032PTA276-1-2, CS 3032PTA276-3-4, and CS 3032PTA137-4-10 genotypes were poorly adapted.

The results of the different methods generally agree, except for the method of Wricke (1965). This method indicated the CS 3032PTA276-3-4, B3PTA382-2-10, and CS 3032PTA137-4-10 genotypes for protein content - which were also indicated by the other methods -, and CD 219RR for oil content, also indicated by the methods of Annicchiarico (1992) and Lin \& Binns (1988).

Sudaric et al. (2006) also observed significant GxE interaction for protein and oil contents, and soybean cultivars with wide adaptability and phenotypic stability for these traits. The significant effects of environments on protein and oil contents were also reported by other authors (Fehr et al., 2003; Ávila et al., 2007; Albrecht et al., 2008).

Based on the estimates of adaptability and stability, and on phenotypic values, the BARC-8, BR 8014887 , and CS 3032PTA276-3-4 genotypes stood out for protein content; whereas Suprema, CD 01RR8384, and A7002 stood out for oil content. The protein and oil contents for these soybean genotypes were higher than the ones found by Fehr et al. (2003), Sudaric et al. (2006), Ávila et al. (2007), and Albrecht et al. (2008).
The Annicchiarico, centroid, and modified Lin \& Binns methods selected similar genotypes, considering high productivity and wide stability.

\section{Conclusion}

The BARC-8, BR 8014887, and CS 3032PTA276-3-4 soybean genotypes are the best parents for increasing protein contents; whereas the Suprema, CD 01RR8384, and A7002 genotypes are the best for increasing oil content.

\section{Acknowledgements}

To Conselho Nacional de Desenvolvimento Científico e Tecnológico (CNPq) and to Fundação de Amparo à Pesquisa do Estado de Minas Gerais (Fapemig), for financial support.

\section{References}

AKOND, A.G.M.; RAGIN, B.; BAZZELLE, R.; KANTARTZI, S.K.; MEKSEM, K.; KASSEM, M.A. Quantitative trait loci associated with moisture, protein, and oil content in soybean [Glycine $\max$ (L.) Merr.]. Journal of Agricultural Science, v.4, p.16-25, 2012.

ALBRECHT, L.P.; BRACCINI, A. de L.A. e; ÁVILA, M.R.; SUZUKI, L.S.; SCAPIM, C.A.; BARBOSA, M.C. Teores de óleo, proteínas e produtividade de soja em função da antecipação da semeadura na região oeste do Paraná. Bragantia, v.67, p.865-873, 2008. DOI: 10.1590/S0006-87052008000400008.

ANNICCHIARICO, P. Cultivar adaptations and recommendation from alfalfa trials in northern Italy. Journal of Genetics and Breeding, v.46, p.269-278, 1992.

ÁVILA, M.R.; BRACCINI, A. de L. e; SCAPIM, C.A.; MANDARINO, J.M.G.; ALBRECHT, L.P.; VIDIGAL FILHO, P.S. Componentes do rendimento, teores de isoflavonas, proteínas, óleo e qualidade de sementes de soja. Revista Brasileira de Sementes, v.29, p.111-127, 2007. DOI: 10.1590/S0101-31222007000300014.

BARBOSA, V. da S.; PELUZIO, J.M.; AFFÉRRI, F.S.; SIQUEIRA, G.B. de. Comportamento de cultivares de soja, em diferentes épocas de semeaduras, visando a produção de biocombustível. Revista Ciência Agronômica, v.42, p.742-749, 2011. DOI: 10.1590/S1806-66902011000300021.

BOERMA, H.R.; SPECHT, J.E. Soybeans: improvement, production and uses. $3^{\text {rd }}$ ed. Madison: American Society of Agronomy, 2004. 1180p.

CRUZ, C.D. Programa Genes: biometria. Viçosa: Ed. da UFV, 2006. 382p.

CRUZ, C.D.; CARNEIRO, P.C.S. Modelos biométricos aplicados ao melhoramento genético. Viçosa: Ed. da UFV, 2003. 585p.

Pesq. agropec. bras., Brasília, v.49, n.6, p.475-482, jun. 2014 DOI: 10.1590/S0100-204X2014000600009 
CRUZ, C.D.; CASTOLDI, F.L. Decomposição da interação genótipos x ambientes em partes simples e complexa. Revista Ceres, v.38, p.422-430, 1991.

CUCOLOTTO, M.; PÍPOLO, V.C.; GARBUGLIO, D.D.; FONSECA JÚNIOR, N. da S.; DESTRO, D.; KAMIKOGA, M.K. Genotype x environment interaction in soybean: evaluation through three methodologies. Crop Breeding and Applied Biotechnology, v.7, p.270-277, 2007. DOI: 10.12702/1984-7033.v07n03a06.

FEHR, W.R.; HOECK, J.A.; JOHNSON, S.L.; MURPHY, P.A.; NOTT, J.D.; PADILLA, G.I.; WELKE, G.A. Genotype and environment influence on protein components of soybean. Crop Science, v.43, p.511-514, 2003. DOI: 10.2135/cropsci2003.0511.

JAUREGUY, L.M.; CHEN, P.; SCABOO, A.M. Heritability and correlations among food-grade traits in soybean. Plant Breeding, v.130, p.647-652, 2011. DOI: 10.1111/j.1439-0523.2011.01887.x.

KAMALUDDIN, M.N.K.; AHMAD, S. Stability analysis of soybean genotypes for yield and yield traits sown under temperate condition in Kashmir Valley. Journal of Food Legumes, v.25, p.175-178, 2012.

LIANG, H.-Z.; YU, Y.-L.; WANG, S.-F.; LIAN, Y.; WANG, T.-F.; WEI, Y.-L.; GONG, P.-T.; LIU, X.-Y.; FANG, X.-J.; ZHANG, M.-C. QTL mapping of isoflavone, oil and protein contents in soybean (Glycine max L. Merr.). Agricultural Sciences in China, v.9, p.1108-1116, 2010. DOI: 10.1016/S1671-2927(09)60197-8.

LIN, C.S.; BINNS, M.R. A superiority measure of cultivar performance for cultivar $\mathrm{x}$ location data. Canadian Journal of Plant Science, v.68, p.193-198, 1988. DOI: 10.4141/cjps88-018.

NAEVE, S.L.; HUERD, S.C. Year, region, and temperature effects on the quality of Minnesota's soybean crop. Agronomy Journal, v.100, p.690-695, 2008. DOI: 10.2134/agronj2007.0204.

NASCIMENTO, M.; CRUZ, C.D.; CAMPANA, A.C.M.; TOMAZ, R.S.; SALGADO, C.C.; FERREIRA, R. de P. Alteração no método centróide de avaliação da adaptabilidade genotípica. Pesquisa Agropecuária Brasileira, v.44, p.263-269, 2009. DOI: 10.1590/ S0100-204X2009000300007.

OLIVEIRA, M.A.R. de; PÍPOLO, V.C.; SCHUSTER, I.; VICENTE, D.; DELLAGOSTIN, M.; OLIVEIRA, E.F. de. Soybean stability and adaptability in Southern and Central Brazil. Crop Breeding and Applied Biotechnology, v.6, p.55-64, 2006. DOI: 10.12702/1984-7033.v06n01a08.

PELÚZIO, J.M.; FIDELIS, R.R.; GIONGO, P.; SILVA, J.C. da; CAPPELLARI, D.; BARROS, H.B. Adaptabilidade e estabilidade de cultivares de soja em quatro épocas de semeadura no sul do Estado do Tocantins. Revista Ceres, v.55, p.34-40, 2008.
POPOVIC, V.; VIDIC, M.; JOCKOVIC, D.; IKANOVIC, J.; JAKSIC, S.; CVIJANOVIC, G. Variability and correlations between yield components of soybean [Glycine max (L.) Merr.]. Genetika, v.44, p.33-45, 2012. DOI: 10.2298/ GENSR1201033P.

PROULX, R.A.; NAEVE, S.L. Pod removal, shade, and defoliation effects on soybean yield, protein, and oil. Agronomy Journal, v.101, p.971-978, 2009. DOI: 10.2134/agronj2008.0222x.

RAY, C.L.; SHIPE, E.R.; BRIDGES, W.C. Planting date influence on soybean agronomic traits and seed composition in modified fatty acid breeding lines. Crop Science, v.48, p.181-188, 2008. DOI: $10.2135 /$ cropsci2007.05.0290.

ROBERTSON, A. The sampling variance of the genetic correlation coefficient. Biometrics, v.15, p.469-485, 1959. DOI: $10.2307 / 2527750$

RODRIGUES, J.I. da S.; MIRANDA, F.D. de; FERREIRA, A.; BORGES, L.L.; FERREIRA, M.F. da S.; GOOD-GOD, P.I.V.; PIOVESAN, N.D.; BARROS, E.G. de; CRUZ, C.D.; MOREIRA, M.A. Mapeamento de QTL para conteúdos de proteína e óleo em soja. Pesquisa Agropecuária Brasileira, v.45, p.472-480, 2010. DOI: 10.1590/S0100-204X2010000500006.

ROTUNDO, J.L.; WESTGATE, M.E. Meta-analysis of environmental effects on soybean seed composition. Field Crops Research, v.110, p.147-156, 2009. DOI: 10.1016/j. fcr.2008.07.012.

SHI, A.; CHEN, P.; ZHANG, B.; HOU, A. Genetic diversity and association analysis of protein and oil content in food-grade soybeans from Asia and the United States. Plant Breeding, v.129, p.250-256, 2010. DOI: 10.1111/j.1439-0523.2010.01766.x.

SILVA, N.R.S.; MARTINS, S.V.; MEIRA NETO, J.A.A.; SOUZA, A.L. de. Composição florística e estrutura de uma floresta estacional semidecidual montana em Viçosa, MG. Revista Árvore, v.28, p.397-405, 2004. DOI: 10.1590/ S0100-67622004000300011.

SUDARIC, A.; SIMIC, D.; VRATARC, M. Characterization of genotype by environment interactions in soybean breeding programmes of southeast Europe. Plant Breeding, v.125, p.191-194, 2006. DOI: 10.1111/j.1439-0523.2006.01185.x.

WRICKE, G. Zur berechnung der okovalenz bei sommerweizen und hafer. Pflanzenzüchtung, v.52, p.127-138, 1965.

ZHE, Y.; LAUER, J.G.; BORGES, R.; LEON, N. Effects of genotype $\mathrm{x}$ environment interaction on agronomic traits in soybean. Crop Science, v.50, p.696-702, 2010. DOI: 10.2135/ cropsci2008.12.0742.

Received on January 22, 2013 and accepted on May 26, 2014

Pesq. agropec. bras., Brasília, v.49, n.6, p.475-482, jun. 2014

DOI: $10.1590 / \mathrm{S} 0100-204 X 2014000600009$ 